\title{
OS RISCOS DOS INIBIDORES DE APETITE: A SIBUTRAMINA
}

\author{
Karinne Nascimento dos Santos ${ }^{1}$ \\ Luciana Ferreira Mattos Colli ${ }^{2}$
}

RESUMO: O objetivo do trabalho é analisar os riscos do inibidor de apetite sibutramina e aatuação do farmacêutico na redução do risco de uso dos inibidores de apetite. A meta no processo de perda de peso é prevenir ou reduzir a morbidade e mortalidade associadas à obesidade, melhorando os fatores de risco cardiovascular e metabólico. Infelizmente, há poucas evidências de que os medicamentos para perda de peso disponíveis atinjam esse objetivo. Recentemente, as preocupações com a segurança do paciente superaram a evidência do benefício de muitos medicamentos para perda de peso. $O$ método de revisão bibliográfica permite incluir pesquisas experimentais e não experimentais, obtendo a combinação de dados empíricos e teóricos que podem direcionar à definição de conceitos, identificação de lacunas nas áreas de estudos, revisão de teorias e análise metodológica dos estudos sobre um determinado tópico. Este método exige recursos, conhecimentos e habilidades para o seu desenvolvimento.

Palavras-chave: Inibidores de apetite. Farmacêutico. Sibutramina. Obesidade. Cardiovascular.

ABSTRACT: The objective of this work is to analyze the risks of the appetite suppressant sibutramine and the role of the pharmacist in reducing the risk of using appetite suppressants. The goal in the weight loss process is to prevent or reduce obesityassociated morbidity and mortality by improving cardiovascular and metabolic risk factors. Unfortunately, there is little evidence that available weight-loss medications achieve this goal. Recently, concerns about patient safetyhave overtaken the evidence for the benefit of many weight-loss medications. The literature review method allows including experimental and non-experimental research, obtaining the combination of empirical and theoretical data that can leadto the definition of concepts, identification of gaps in the areas of study, review of theories and methodological analysis of studies on a particular topic. This method requires resources, knowledge and skills for its development.

Keywords: Appetite suppressants. Pharmaceutical. Sibutramine. Obesity. Cardiovascular.

\footnotetext{
${ }^{\mathrm{I}}$ Universidade Iguaçu.

${ }^{2}$ Professora orientadora do curso de Farmácia pela. Universidade Iguaçu. Formação acadêmica: Ensino superior em Farmácia/ Universidade Iguaçu
} 


\title{
INTRODUÇÃO
}

Durante os últimos 50 anos, a pandemia global de obesidade e comorbidades associadas com risco de vida promoveram fortemente o desenvolvimento da farmacoterapia anti-obesidade.

\begin{abstract}
A sibutramina é uma droga anti-obesidade que, em conjunto com as modificações no estilo de vida, reduz a ingestão de alimentos e o peso corporal. Isso pode resultar de vários efeitos: inibição da recaptação présináptica de neurotransmissores monoaminérgicos no sistema nervoso central, suprimindo assim o apetite, indução de um aumento de anorexígenos e uma diminuição da secreção de neuropeptídeos orexigênicos, indução de um aumento no gasto de energia e indução de efeitos simpaticomiméticos (MARINI, SILVA, OLIVEIRA 2014).
\end{abstract}

Os efeitos da sibutramina nos sinais anabólicos e catabólicos que regulam a homeostase energética no hipotálamo não são completamente compreendidos. Apesar de ser uma droga útil no tratamento da obesidade, surgiu recentemente a consciência sobre a perda de eficácia em longo prazo e os efeitos colaterais prejudiciais da sibutramina. Como consequência, novos medicamentos que produzem uma perda de peso mais segura e persistente estão atualmente em testes clínicos (GONZAGA, et al., 2015).

Sibutramina é eficaz na promoção da perda de peso. A perda de peso com a sibutramina está associada a mudanças positivas e negativas nos fatores de risco cardiovascular e metabólico. Não há evidências suficientes

para determinar com precisão o perfil de risco-benefício de longo prazo para a sibutramina (FRANCO, COMINATO, DAMIANI 2014).

O National Institutes of Health publicou diretrizes para o tratamento da obesidade, que indicam que todos os adultos obesos (índice de massa corporal, 230) e todos os adultos com índice de massa corporal de pelo menos 27 e fatores de risco ou doenças concomitantes são candidatos à terapia medicamentosa (CASSIN, 2018). 
Dados esses critérios amplos, mais de roo milhões de adultos podem ser elegíveis para receber terapia medicamentosa para obesidade nos Estados Unidos. As diretrizes do National Institutes of Health são baseadas em um crescente corpo de evidências que relaciona a obesidade adulta com o aumento do risco de várias condições crônicas, redução da qualidade de vida emortalidade precoce (CAMPOS, et al., 2014).

O objetivo aceito de perda de peso é prevenir ou reduzir a morbidade e mortalidade associadas à obesidade, melhorando os fatores de risco cardiovascular e metabólico. Infelizmente, há poucas evidências de que os medicamentos para perda de peso disponíveis atinjam esse objetivo. Recentemente, as preocupações com a segurança superaram a evidência do benefício de muitos medicamentos para perda de peso. O objetivo do trabalho é analisar os riscos dos inibidores de apetite sibutramina e a atuação do farmacêutico nos perigos dos inibidores de apetite.

\section{MODULAÇÃO NEUROENDÓCRINA DO APETITE}

O apetite é o desejo de ingestão de alimentos. Sua regulação depende da integração e transdução de sinais episódicos e tônicos nos centros reguladores de energia do SNC localizados no hipotálamo, particularmente no núcleo arqueado (ARC). Os sinais episódicos são entradas de curto prazo geradas antes da ingestão da refeição, enquanto os sinais tônicos são entradas de curto ou longo prazo geradas pelo consumo, absorção e metabolismo dos alimentos e estoques de energia corporal (MUSTEIKIS, 2015). 
Figura r: MODULAÇÃO NEUROENDÓCRINA DO APETITE

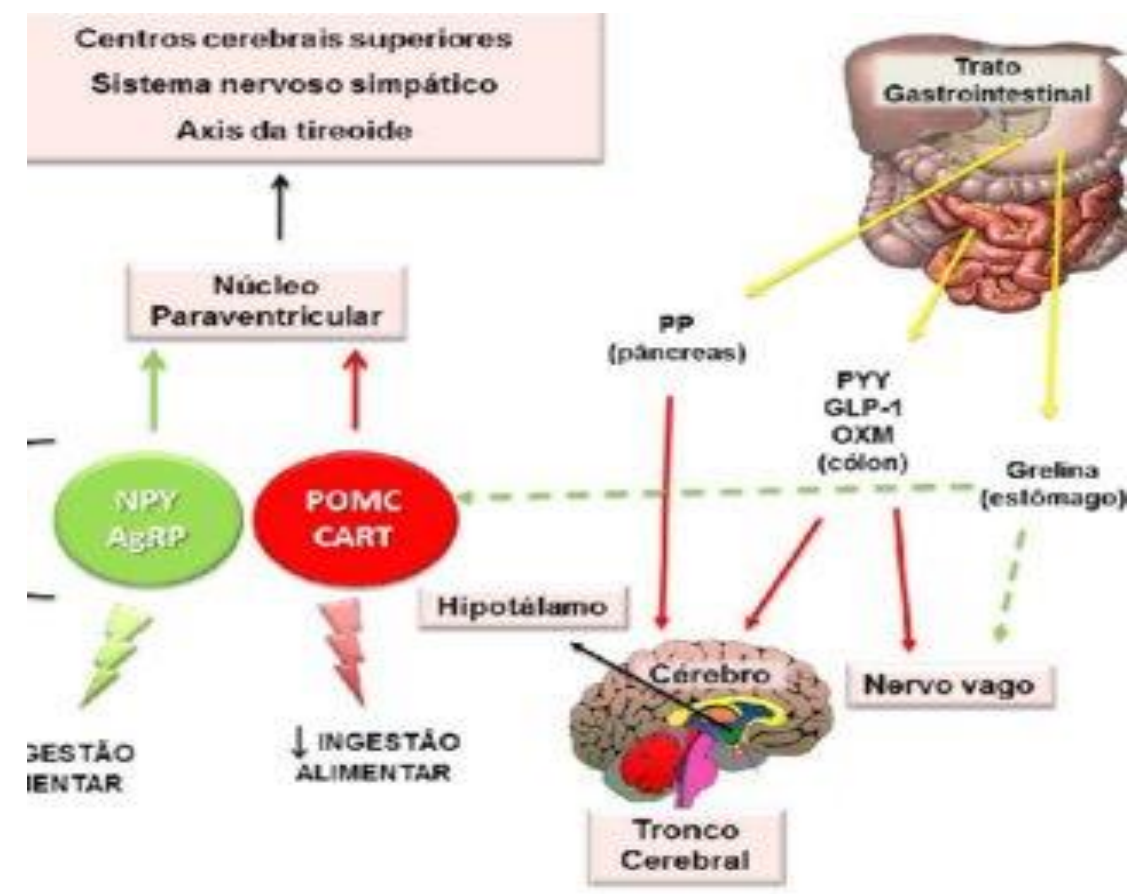

Fonte: (MUSTEIKIS, 2015).

Ambos podem ser anabólicos (estimulando a ingestão de energia e suprimindo o gasto de energia) ou catabólicos (inibindo a ingestão de energia e estimulando o gasto de energia). Os sinais tônicos e episódicos podem ser divididos em três categorias:

I) sinais originados da estimulação de receptores no intestino e de alterações metabólicas no fígado e tecido adiposo, que são transmitidos viasinais vagais aferentes ou hormônios para receptores localizados no ARC e no núcleo do complexo trato solitário / área postrema no tronco encefálico. Estes podem ser sinais de saciedade (por exemplo, peptídeos gastrointestinais anabólicos e catabólicos como grelina e colecistoquinina, respectivamente) e sinais de adiposidade (por exemplo, leptina e insulina);

2) nutrientes e precursores de neurotransmissores que cruzam a barreira hematoencefálica alterando a atividade neuroquímica do SNC, e

3) em resposta a essas duas entradas, sinais eferentes que surgem de receptores 
localizados no SNC que detectam níveis circulantes de nutrientes, hormônios e outrosfatores da periferia (CAMPOS, et al., 2014). Esses sinais

eferentes incluem numerosos neuropeptídeos orexigênicos $e$ anorexigênicos $e$ neurotransmissores monoaminérgicos, que estão contidos em neurônios que se projetam do ARC para áreas a jusante envolvidas na percepção da saciedade núcleos ventromediais e núcleos paraventriculares (PVN) e fome área hipotalâmica lateral (LHA). A saída líquida do PVN é catabólica, aumentando a potência dos sinais de saciedade, enquanto a saída líquida do LHA é, por contraste, anabólica, suprimindo a atividade dos sinais de saciedade. Em conjunto, as flutuações nos sinais episódicos e tônicos determinam fortemente o apetite, a fome e a saciedade, sendo, portanto, essenciais para a regulação do peso corporal (CASSIN, 2018).

\section{METODOLOGIA}

O tipo do estudo é uma revisão bibliográfica, pesquisas do tipo tem o objetivo primordial à exposição dos atributos de determinado fenômeno ou afirmação entre suas variáveis (GIL, 2018). Assim, recomenda-se que apresente características do tipo: analisar a atmosfera como fonte direta dos dados e o pesquisador como um instrumento interruptor; não agenciar o uso de artifícios e métodos estatísticos, tendo como apreensão maior a interpretação de fenômenos e a imputação de resultados, o método deve ser o foco principal para a abordagem e não o resultado ou o fruto, a apreciação dos dados deve ser atingida de forma intuitiva e indutivamente através do pesquisador (GIL, 2018).

O método de revisão bibliográfica permite incluir pesquisas experimentais e não experimentais, obtendo a combinação de dados empíricos e teóricos que podem direcionar à definição de conceitos, identificação de lacunas nas áreas de estudos, revisão de teorias e análise metodológica dos estudos sobre um determinado tópico. Este método exige recursos, conhecimentos e habilidades para o seu desenvolvimento (GIL, 2018).

Considerando a classificação proposta por Gil (2018, p. 5), pode-se afirmar que "esta proposta é melhor representada por meio de uma pesquisa do tipo exploratória, cujo objetivo é possibilitar um maior conhecimento a respeito do problema, de modo a torná- 
lo mais claro ou auxiliando na formulação de hipóteses". No entendimento do autor, o principal objetivo deste tipo de pesquisa pode ser tanto o aprimoramento de ideias, quanto a descoberta de intuições, o que o torna uma opção bastante flexível, gerando, na maioria dos casos, uma pesquisa bibliográfica ou um estudo de caso. (GIL, 2018).

O desenvolvimento dessa revisão integrativa foi fundamentado conforme as seis etapas propostas por Gil (2018). São elas: I. Identificação do tema e formulação da questão norteadora; 2. Definição dos critérios de inclusão e exclusão; 3. Definição das informações que serão extraídas dos estudos; 4. Avaliação dos estudos; 5. Interpretação dos resultados; 6. Apresentação da revisão do conhecimento.

Esta etapa foi representada pelo estabelecimento de critérios para inclusão e exclusão de estudos/ amostragem ou busca na literatura. Para a busca dos artigos foram utilizadas as bases de dados: Biblioteca Virtual em Saúde (BVS), Literatura LatinoAmericana e do Caribe e Ciências da Saúde (LILACS) e na Medical Literature Analysis and Retrieval Sistem on-line (MEDLINE) e Scientific Electronic Library Online (SciELO).

As estratégias de busca foram efetivadas, via filtros de busca SCIELO e BIREME (LILACS e MEDLINE, utilizando os descritores de saúde: "Inibidores de apetite, Farmaceutico; Sibutramina”.

Como critérios de inclusão foi considerado todos os artigos publicados nas bases de dados informadas, dentro da temporariedade prevista de 2013 a 2020 com texto completo disponível, publicados em revistas de revisões de literatura indexadas e no idioma português e inglês. Critérios de exclusão foram exclusos os artigos não relacionados ao tema; artigos de opinião; relatórios; editoriais; enfim, literatura cinzenta. Artigos duplicados nos bancos de dados foram consideradas uma única versão para a análise, artigos publicados fora do tempo estabelecido e/ou que não contenha o texto na integra.

Nessa etapa é importante ter a busca nas bases de dados deve ser ampla e diversificada. O ideal é que todos os artigos encontrados sejam utilizados e os critérios de amostragem precisam garantir a representatividade da amostra, sendo importantes indicadores da confiabilidade e da fidedignidade dos resultados (GIL, 2018). 


\section{RESULTADOS E DISCUSSÃO}

A sibutramina, com os nomes comerciais Meridia $^{\circledR}$ e $\operatorname{Reductil}^{\circledR}$, é um medicamento administrado por via oral atualmente aprovado nos Estados Unidos da América, e até recentemente também aprovado na Europa, para o tratamento de longo prazo da obesidade. É uma $\beta$-fenetilamina que, quando administrada em doses de 5-15 mg / dia e em conjunto com terapia de estilo de vida (dieta calórica reduzida, exercício físico e terapia comportamental alimentar), pode induzir um modesto, mas significativo, 5-10 \% de perda de peso corporal $(4-8 \mathrm{~kg})$ na maioria dos pacientes obesos e com sobrepeso (CASSIN, 2018).

A capacidade da sibutramina de reduzir o peso corporal pode ser explicada por vários motivos. Em primeiro lugar, seu efeito mais bem descrito é uma inibição seletiva da recaptação pré-sináptica dos neurotransmissores monoaminérgicos serotonina (5hidroxitriptamina; $5-\mathrm{HT}$ ), noradrenalina (NA) e, em menor extensão, dopamina, no nível do SNC, aumentando o apetite suprimindo ações desses neurotransmissores (GONZAGA, et al., 2015).

A recaptação desses neurotransmissores da sinapse via alta afinidade e transportadores específicos presentes na membrana pré-sináptica (que são inibidos pela sibutramina), termina sua sinalização. Ao contrário das primeiras drogas semelhantes às anfetaminas, como dexanfetamina, fentermina e fenfluraminas, a sibutramina não estimula diretamente a liberação de $5-\mathrm{HT}$, NA nem dopamina e, portanto, não causa depleção de neurotransmissores pré-sinápticos nem a consequente neurotoxicidade. Além disso, há evidências de que a sibutramina não se liga a nenhum dos receptores póssinápticos serotoninérgicos, adrenérgicos ou dopaminérgicos (MARINI, SILVA, OLIVEIRA 2014).

Em segundo lugar, a sibutramina aumenta a atividade anorexigênica e reduz a liberação de neuropeptídeos orexígenos no ARC. Estudos em modelos animais têm contribuído para melhor elucidar esse efeito. De fato, em ratos com restrição de energia, o tratamento com sibutramina aumentou o transporte do hormônio anorexigênico leptina para o ARC, que por sua vez ativou a proopiomelanocorticotropina (POMC) / cocaína anfetamina regulando os neurônios (CART) e inibiu o neuropeptídeo Y (NPY) / peptídeo relacionado à agouti 
Revista Ibero- Americana de Humanidades, Ciências e Educação- REASE

(AgRP) neurônios (MUSTEIKIS, 2015).

Consequentemente, isso estimulou a secreção dos neuropeptídeos anorexigênicos POMC e seu derivado hormônio estimulador de melanocortina $\alpha$ melanócito ( $\alpha-\mathrm{MSH})$, e do hormônio liberador de corticotrofina (CRH) e CART. O $\alpha$ MSH liberado pode se ligar e ativar os receptores de melanocortina (MCR), particularmente MCR-4, no PVN. Ao contrário, a secreção dos neuropeptídeos orexigênicos NPY, AgRP, orexina A e B e hormônio concentrador de melanocortina $(\mathrm{MCH})$ é inibida (CASSIN, 2018).

A capacidade da sibutramina de diminuir o peso corporal também pode ser atribuída ao fato de aumentar o gasto de energia. Isso pode ser alcançado por dois efeitos distintos. Em primeiro lugar, a sibutramina previne a diminuição do gasto energético basal que segue a perda de peso, um efeito que pode ser explicado pela ativação do MCR4. Em segundo lugar, a sibutramina pode aumentar a termogênese por meio da ativação do receptor $\beta_{3}$-adrenérgico, um efeito mediado por NA, no tecido adiposo branco periférico. Além disso, em ratos, mas não em humanos, a sibutramina parece induzir hipofagia por meio de efeitos simpaticomiméticos, envolvendo regulação negativa de $\beta_{\mathrm{I}}$ pré e pós-sináptico- e receptores $\alpha_{2}$-adrenérgicos e $5-\mathrm{HT}_{\mathrm{IA}}$ localizados no $\mathrm{PVN}$ (FRANCO, COMINATO, DAMIANI 2014).

Esses efeitos são conhecidos por estarem associados a uma ação antidepressiva, o que explica porque a sibutramina foi originalmente desenvolvida como um medicamento antidepressivo. No entanto, a sibutramina é hoje utilizada como agente supressor do apetite, sendo até recentemente o mais prescrito. Os efeitos de redução de peso da sibutramina são amplamente atribuídos aos seus metabólitos de amina primários ( $\mathrm{N}$ desmetilsibutramina; BTS 54505) e secundários (N-didesmetilsibutramina; BTS 54 354), e não ao composto original (GONZAGA, et al., 2015).

Normalmente, 6 meses após o início do tratamento com sibutramina, a ingestão de alimentos se recupera e o peso corporal estabiliza até que a sibutramina seja descontinuada. Este fenômeno é denominado taquifilaxia da sibutramina. Após a descontinuação, a recuperação do peso perdido é freqüentemente observada. Isso destaca que a sibutramina é eficaz na redução do peso corporal e da ingestão de alimentos quando 
administrada por curtos períodos de tempo (6-12 meses), mas sua eficácia diminui quando administrada cronicamente (> I ano) (CAMPOS, et al., 2014).

De fato, em humanos, não há estudos de mais de 2 anos avaliando os efeitos da sibutramina na redução de peso. A taquifilaxia com sibutramina pode ser explicada por mecanismos homeostáticos contra-reguladores que promovem resistência a futuras ações de redução de peso dessa droga. Esses mecanismos contra-reguladores podem ser explicados a seguir (FRANCO, COMINATO, DAMIANI 2014).

A administração de sibutramina em curto prazo reduz o ponto de ajuste do peso corporal. Este ponto de ajuste de peso corporal não é permanentemente reduzido pela administração de sibutramina a longo prazo, uma vez que o cérebro se oporá a essa mudança estimulando o apetite, recompensa alimentar (prazer associado ao consumo de um alimento saboroso) e alimentos ingestão e também diminuindo o gasto de energia basal e diminuindo as reduções nos estoques de gordura corporal (CAMPOS, et al., 2014).

Musteikis, (2015), aponta que a diminuição dos níveis circulantes de leptina no sangue que acompanha a redução da gordura corporal pode desencadear mecanismos contrários-reguladores que bloqueiam os efeitos da sibutramina na perda de peso, pois descobriram que a restauração dos níveis circulantes de leptina durante o longo prazo (8 semanas) A terapia com sibutramina diminuiu o peso corporal e a ingestão de alimentos e aumentou a oxidação de gordura em ratos obesos.

Apesar da eficácia de curto prazo na redução do peso corporal e da ingestão de alimentos, a sibutramina tem alguns efeitos colaterais indesejáveis que podem ou não estar diretamente relacionados com seu efeito antiobesidade. Os principais efeitos colaterais da sibutramina estão associados à estimulação noradrenérgica dos circuitos do apetite hipotalâmico e às propriedades simpatomiméticas inerentes. Boca seca, dor de cabeça, insônia, astenia, obstipação e em alguns casos amnésia são alguns dos efeitos colaterais (GONZAGA, et al., 2015).

A sibutramina também pode induzir mudanças de humor porque o controle da homeostase energética e do humor geralmente usa circuitos cerebrais sobrepostos (por exemplo, o sistema serotoninérgico). Ao contrário de outras drogas supressoras do apetite (por exemplo, fentermina e dexanfetamina) e apesar de ser estruturalmente semelhante à anfetamina, a sibutramina não tem potencial de abuso, uma vez que não aumenta a 
liberação de dopamina na sinapse. Devido a um aumento do impulso simpático para o sistema CDV, a sibutramina pode aumentar a pressão arterial sistólica e / ou diastólica (2-20 $\mathrm{mm} \mathrm{Hg})$ e a frequência cardíaca (3-20 batimentos / min) (MARINI, SILVA, OLIVEIRA 2014).

De acordo com esses efeitos, o Sibutramine in Cardiovascular Outcomes Trial (SCOUT) descobriu que em indivíduos obesos e com sobrepeso, a sibutramina aumentou a morbidade da doença de CDV (enfarte do miocárdio ou acidente vascular cerebral), levando a Agência Europeia de Medicamentos a retirar este medicamento do mercado em 2010. Portanto, por causa de seus efeitos colaterais cardiovasculares inerentes, a sibutramina não pode ser usada para testar a hipótese de que a perda de peso pode diminuir o risco cardiovascular. $\mathrm{Na}$ Europa, isso deixa apenas um medicamento aprovado, Orlistat ${ }^{\circledR}$, para o tratamento de longo prazo da obesidade (FRANCO, COMINATO, DAMIANI 2014).

\section{ATUAÇÃO DO FARMACÊUTICO E OS INIBIDORES DE APETITE}

Os farmacêuticos são muitas vezes o primeiro profissional de saúde que uma pessoa com uma alimentação e a desordem. Os farmacêuticos podem desempenhar um papel vital na detecção de transtornos alimentares, monitorando o uso de medicamentos prescritos e não prescritos medicamentos e produtos. Como farmacêutico, está em uma posição fundamental para detectar a presença de um transtorno alimentar, incentive a busca de ajuda e auxilie o paciente com referências (CAMPOS, et al., 2014).

Os profissionais de saúde podem desempenhar um papel importante na educação de pacientes com sobrepeso e obesos sobre as estratégias ideais para redução de peso e sobre os riscos e benefícios dos medicamentos para emagrecer. Os farmacêuticos são considerados um dos profissionais de saúde mais acessíveis e confiáveis (CASSIN, 2018).

Muitos pacientes que desejam perder peso visitam a farmácia comunitária para obter produtos de controle de peso ou buscar aconselhamento de saúde. Além disso, dispositivos de ponto de atendimento comumente usados para controle de peso estão se tornando prontamente disponíveis nas farmácias comunitárias (FRANCO, COMINATO, DAMIANI 2014). 
Figura 2: FARMACÊUTICO E OS INIBIDORES DE APETITE

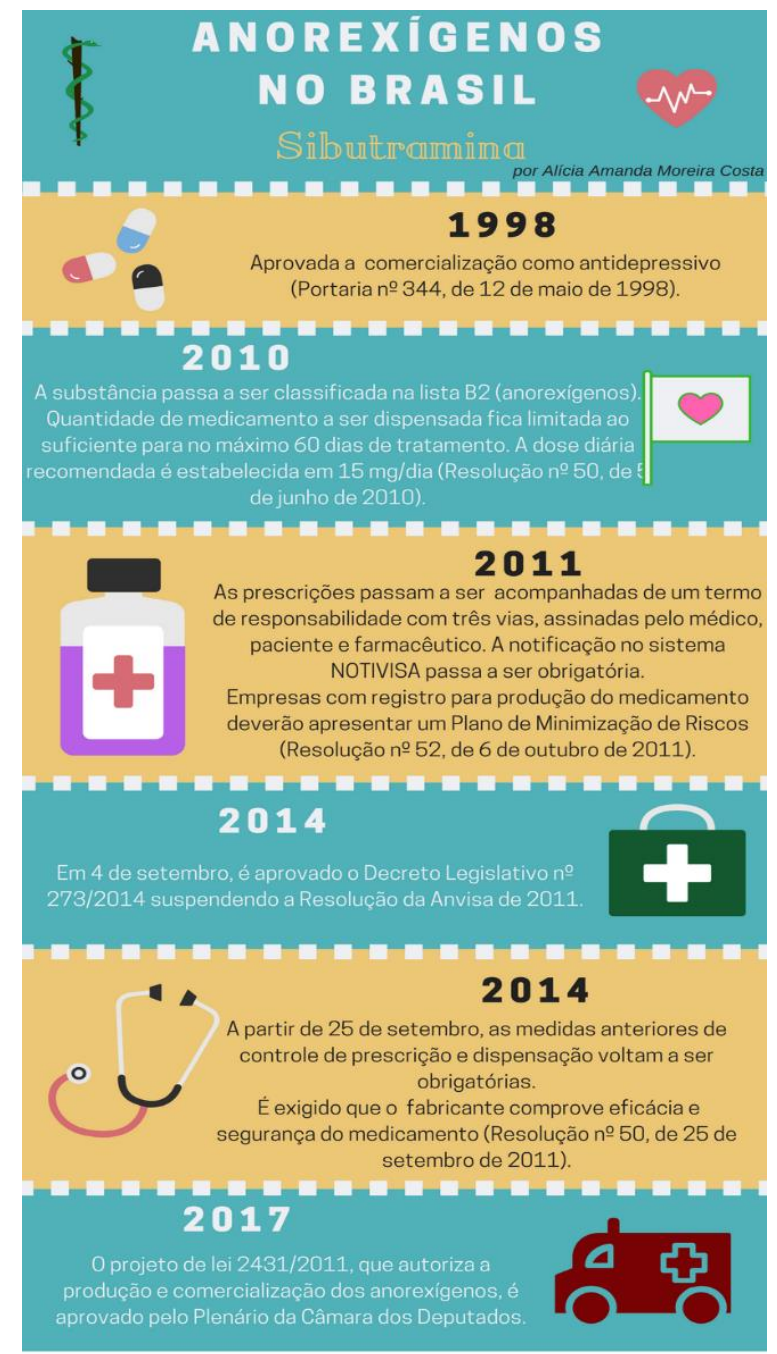

Para saber mais sobre o assunto, leia:

"Anorexígenos e sibutramina... uma discussão complexa e uma decisão

perigosa", no blog do Cemed.

Fonte: (CASSIN, 2018).

Os farmacêuticos podem intervir ativamente e desempenhar um papel fundamental no controle da obesidade. No nível mais baixo de engajamento, eles podem ajudar os pacientes a selecionar um medicamento apropriado para emagrecer e aconselhálos sobre o uso apropriado de medicamentos para emagrecer. No nível mais alto de envolvimento, os farmacêuticos sozinhos ou em colaboração com médicos e nutricionistas podem fornecer serviços avançados de controle de peso (GONZAGA, et al., 2015). 
Na prestação desses serviços, o farmacêutico poderia usar dispositivos de ponto de atendimento, revisar o histórico de saúde do paciente, avaliar a prontidão do paciente para mudar um comportamento de saúde indesejável, oferecer aconselhamento comportamental motivacional, projetar um plano farmacoterapêutico e monitorar a segurança e eficácia dos medicamentos para emagrecer (MARINI, SILVA, OLIVEIRA 2014).

No entanto, permanece o fato de que os inibidores de apetite não são medicamentos prescritos. Os suplementos vendidos ao balcão para suprimir o apetite não são regulamentados e são potencialmente perigosos. Também não há dados médicos ou clínicos para mostrar que funcionam, apesar de qualquer endosso qualitativo. Existem muitos efeitos colaterais indesejáveis para os supressores, e os supressores não regulamentados são potencialmente perigosos (MUSTEIKIS, 2015).

Supressores de apetite podem ajudar a suprimir a fome e fazer com que você se sinta saciado mais cedo, portanto, podem ajudá-lo a perder peso em curto prazo. No entanto, existem maneiras mais seguras de perder peso. Os perigosos efeitos colaterais das pílulas dietéticas no passado fizeram com que certas marcas fossem proibidas no Reino Unido e nos Estados Unidos. Há um comércio florescente de pílulas dietéticas na Internet que tem permitido que pessoas desesperadas para perder peso comprem drogas ilegais e potencialmente perigosas e suplementos de ervas (CAMPOS, et al., 2014).

\section{CONCLUSÃO}

A sibutramina é uma droga anti-obesidade que produz reduções modestas e de curto prazo do peso corporal, principalmente devido ao seu efeito supressor do apetite. Apesar de ser uma droga útil no tratamento da obesidade, surgiu recentemente a consciência sobre a perda de eficácia e os efeitos colaterais prejudiciais da sibutramina. Como consequência, a identificação de novos fármacos que induzam perda de peso de forma segura e persistente é atualmente um campo de intensa pesquisa.

Drogas que têm como alvo a liberação de neuropeptídeos orexigênicos e anorexigênicos, como agonistas de MCR-4, antagonistas de receptor de MCH e antagonistas de NPY, e outras que têm 
como alvo receptores de neurotransmissores monoaminérgicos, como agonistas de receptor de serotonina $5-\mathrm{HT}{ }_{2} \mathrm{C}$, estão, entre outros, atualmente em ensaios clínicos.

A verdade é que as pessoas em todo o mundo gastam bilhões de dólares em pílulas dietéticas que não funcionam. Esta não é uma boa notícia para pessoas desesperadas por soluções, e há mais notícias ruins. Experimentar novos comprimidos pode ser caro, além de perigoso para a saúde. Muitas pílulas dietéticas mal regulamentadas ou recentemente retiradas estão por aí e consumi-las pode ter efeitos negativos. No pior dos casos, eles podem ser fatais. Comprimidos de dieta perigosos devem ser levados a sério e devem ser evitados em todas as situações. Os riscos não compensam os benefícios.

\section{REFERÊNCIAS BIBLIOGRÁFICAS}

CAMPOS Larissa Soares, OLIVEIRA Lorena Amaral de, DA SILVA Paula Karolinne Pires, PAIVA Andres Marlo Raimundo. Estudo dos efeitos da sibutramina. Rev. Uningá. 2014; v.20 n.3; 50-53.

CASSIN, Jéssica Cristina Dambros. Uso Indiscriminado da Sibutramina como anorexígeno. 2018. Trabalho de Conclusão de Curso (Graduação em Farmácia) Universidade de Cuiabá, Cuiabá, 2018.

DUTRA, Josileyde Ribeiro; SOUZA, Sonia Maria da Fonseca; PEIXOTO, Mariana Chiesa. A influência dos padrões de beleza veiculados pela mídia, como fator decisório na automedicação com moderadores de apetite por mulheres no município de Miracema-RJ. Transformar, [s.1.], v. 7, p.194-213, 2015.

FRANCO R.C, COMINATO L, DAMIANI D. O efeito da sibutramina na perda de peso de adolescentes obesos. Arq. Bras. Endocrinologia \& Metabologia. 2014; v.58, n.3; p.243-250.

GIL, A. C. Como elaborar projetos de pesquisa. 5. ed. São Paulo: Atlas, 2018.

GONZAGA, Josyanne Barros et al. Análise das prescrições de sibutramina dispensadas em drogarias no município de Cuiabá-MT, Brasil. Infarma: Ciências Farmacêuticas, v. 27 , n. I, p.33-37, 30 mar. 2015 .

MARINI Danyelle Cristine, SILVA Leandro de Oliveira, OLIVEIRA Débora Cristina. Perfil da dispensação e do uso de sibutramina para tratamento da obesidade. Foco: Caderno de Estudos e Pesquisas. 2014; 5(7): 6I-78. 
MUSTEIKIS, Ana Carolina. Revisão Sistemática Na Farmacoterapia Da Obesidade: Uma Avaliação Da Eficácia Da Sibutramina. Conic Semesp $15^{\circ}$ Congresso Nacional de Iniciação Cientifica 2015.

RADAELLI Marqueli, PEDROSO Roberto Costa, MEDEIROS Liciane Fernandes. Farmacoterapia da obesidade: Benefícios e Riscos. Saúde e Desenv. Humano. 2016; 4(I): IOI-IIS. 